\title{
Relação materno-filial da raça Morada Nova recebendo dietas com três níveis de energia, ao final da gestação ${ }^{1}$
}

\author{
Tobyas Maia de Albuquerque Mariz ${ }^{2}$, Edgard Cavalcanti Pimenta Filho ${ }^{3}$, Ariosvaldo Nunes de \\ Medeiros $^{3}$, Severino Gonzaga Neto ${ }^{3}$, Saulo Vilarim de Farias Leite ${ }^{4}$, Jacira Neves da Costa Torreão ${ }^{3}$ \\ 1 Parte da dissertação de Mestrado do Primeiro Autor/PPGZ/CCA/UFPB. Financiado FUNDECI/ETENE/BNB. \\ 2 Programa de Doutorado Integrado em Zootecnia/CCA/UFPB. \\ ${ }^{3}$ Departamento de Zootecnia do CCA/UFPB. \\ ${ }^{4}$ EMEPA/PB.
}

RESUMO - Objetivou-se com este trabalho avaliar os efeitos de diferentes níveis de energia ofertados no terço final da gestação sobre o comportamento materno-filial de animais da raça Morada Nova. O experimento consistiu de três tratamentos - três grupos de oito matrizes, alimentadas com dietas isoprotéicas contendo diferentes níveis energéticos (2,0; 2,4 e 2,8 Mcal EM/kg MS), com oito repetições cada. Observou-se efeito dos tratamentos sobre as variáveis comportamentais maternas avaliadas (vocalização, ato de cheirar e lamber a cria e a posição da ovelha - em pé ou deitada), nas ovelhas, mas não foi observada diferença para os comportamentos filiais (posição da cria, tempo decorrido do nascimento à primeira vez que ficou de pé, tempo decorrido do nascimento à primeira mamada, tempo total de mamadas) dos cordeiros. A mobilização de reservas ou o carreamento direto de nutrientes da dieta para o crescimento fetal normal, nos grupos alimentos com dietas contendo 2,0 e 2,4 Mcal EM/kg MS, minimizaram os possíveis efeitos sobre o comportamento filial dos cordeiros. Mesmo sob interferência no relacionamento com as crias, ovelhas Morada Nova conseguiram manter as condições dos cordeiros inalteradas em todos os tratamentos.

Palavras-chave: comportamento ovino, manejo de cordeiros e ovelhas, manejo nutricional

\section{Ewe-lambs relationship in Morada Nova breed under three energy levels at the end of gestation}

\begin{abstract}
The present work was carried out with the objective to evaluate the effects of different energy levels offered in the final third of gestation on the ewe-lambs behavior of animals from the Morada Nova breed. The experiment was divided into three treatments - three groups of eight ewes, fed isoprotein diets containing different energy levels (2.0, 2.4, and 2.8 Mcal ME/kg DM), with eight replicates each. There was effect of the treatment on the maternal behavior evaluated variables (vocalization, act of smell and lick the lambs and the position of the ewe - stand up or lying), in the ewes, but no difference was observed for the filial behavior (position of animal, time from the birth to the first time that the animal was up, time from the birth to the first sucking and total time sucking) of the lambs. The mobilization of reserves or the direct carrying of nutrients of the diet for normal fetal growth, in the groups fed with diets containing 2.0 and $2.4 \mathrm{Mcal} \mathrm{ME} / \mathrm{kg} \mathrm{DM}$, minimized the possible effects on the filial behavior of lambs. The mobilization of reserves or direct carry of nutrients of the diet for normal fetal growth, in the groups fed with diets containing 2.0 and 2.4 Mcal ME $/ \mathrm{kg}$ DM, minimized the possible effect on the filial behavior of lambs. Despite the interference in their relationship with the kids, ewes Morada Nova attained to keep the conditions of the lambs unchanged in all treatments.
\end{abstract}

Key Words: lamb and ewe management, nutritional management, sheep behavior

\section{Introdução}

O estabelecimento da relação entre a mãe e a cria ocorre nas primeiras horas pós-parto (Ramírez et al., 1996), período considerado crítico, pois possibilita o desenvolvimento e a manutenção do comportamento materno-filial. Em cabras e ovelhas, alguns minutos de contato garantem essa ativação, mantendo-se mesmo após uma separação forçada; o vínculo é individualizado e estabelecido por experiências vividas durante a gestação, o parto e os contatos iniciais com as crias (Costa \& Cromberg, 1998). O conjunto de comportamentos para chamar atenção, incluindo cheirar, lamber e proteger o neonato, serve como instrumento para formação de um laço materno-filial seletivo (Alexander et al., 1974; Pinheiro et al., 1997). 
Segundo Brown (1998), entre os inúmeros mecanismos disponíveis para essa ativação, a estimulação vaginocervical, ocorrida durante a passagem do feto pelo canal do parto, é o primeiro evento físico importante. Este mecanismo dá início a uma cadeia de transmissões de estímulos nervosos e hormonais que induz o ato de lamber e cheirar o líquido amniótico que envolve o neonato após o parto, reduzindo também o comportamento agressivo da mãe com os cordeiros.

De acordo com Poindron et al. (1988), o segundo evento fisiológico mais importante durante a parição é a excitação mecânica da área genital causada pela expulsão final do feto, ao qual se associa uma curta, porém importante, liberação de ocitocina.

A manutenção do comportamento maternal é promovida pela ação hormonal (estradiol, prolactina, ocitocina e opióides) e sensorial (audição, olfato e visão). O olfato é extensivamente usado em muitos aspectos maternais em mamíferos, assegurando a coordenação das interações materno-filiais e, por conseguinte, o desenvolvimento normal da descendência. Fora do período de parição e lactação, componentes olfatórios exercem um papel de inibição da responsabilidade materna, fazendo com que as fêmeas nãoprenhes, ou em estádio inicial de gestação, considerem aversivo o odor de um jovem (Levy et al., 2004).

Na hora do parto, entretanto, ocorre uma troca no valor dos odores infantis, de forma que a cria se torna um estímulo muito potente, tornando este processo sensorial parte importante do sistema motivacional da atividade materna. A estrutura neural do bulbo olfatório principal sofre mudanças profundas quando exposta a odores do parto. Estas mudanças contribuem para a responsabilidade materna e memorização dos odores, passando a ser uma importante base para reconhecimento individual pelas mães e para regulação de vários aspectos do comportamento materno (Poindron et al., 1993; Keller et al., 2003).

A remoção dos fluidos pela mãe, minutos após o parto, pode ajudar a reduzir a perda de calor e estimular a atividade de busca da teta pela cria, por meio de movimentos exploratórios no corpo da mãe (visão e audição), que culminarão com a localização do úbere, pela detecção de odores característicos produzidos pelas glândulas inguinais e pelos restos placentários presos à ovelha (Vince, 1993; Schaal et al., 1995).

A inadequada nutrição durante a gestação pode comprometer o desenvolvimento fetal, além de interferir na interação mãe-cria, visto que se observa a rejeição de cordeiros nascidos de ovelhas que apresentam condições corporais inadequadas no momento do parto, potencializando a mortalidade neonatal (Thomson \& Thomson, 1949; Langeneau \& Lerg, 1976; Putu et al., 1988). Durante o terço final da gestação, ocorre em torno de 90\% do desenvolvimento do feto e de seus envoltórios (Ferrel, 1992) e, por esse motivo, observa-se aumento nas exigências nutricionais das matrizes durante este período (Silva Sobrinho et al., 1996; Cunha et al., 1999). O não atendimento dessas exigências pode acarretar diminuição de até $40 \%$ do peso total no desenvolvimento fetal (Mellor, 1987). Segundo Geraseev et al. (2006), a restrição nutricional na vida uterina pode comprometer a produtividade de cordeiros após o desmame, mesmo com aleitamento ad libitum durante a fase materna dependente, reflexo do baixo peso ao nascimento.

Objetivou-se com este trabalho avaliar os efeitos de diferentes níveis de energia ofertados no período gestacional sobre o comportamento materno-filial de ovinos da raça Morada Nova.

\section{Material e Métodos}

O trabalho foi desenvolvido na Estação Experimental de São João do Cariri, pertencente ao Centro de Ciências Agrárias (CCA) da Universidade Federal da Paraíba (UFPB), localizada no município de São João do Cariri-PB.

Os animais ficaram alojados em um conjunto de baias individuais (1,5 m x 2,5 m), dotadas de bebedouro e comedouro, dispostas em fileiras duplas separadas por um corredor.

Durante a fase pré-experimental, foram utilizadas, inicialmente, 37 ovelhas da raça Morada Nova, pertencentes ao rebanho da UFPB, todas pluríparas e vazias. Após a seleção, as ovelhas foram everminadas e submetidas a um período de restrição alimentar, objetivando-se o nivelamento do escore corporal com valor 2,0 (escala de 1 a 5), obtido pela reduzida cobertura vegetal disponível aos animais no piquete.

Após o ajuste do escore corporal, foi utilizado um flushing alimentar, com duração de quatro semanas (duas antes do início do período de acasalamento e duas após este período), associado ao efeito macho, com a finalidade de estimular e sincronizar o cio.

Uma vez detectado o cio, a ovelha era transferida para a baia do reprodutor até que fosse coberta. Caso permanecesse no cio após um intervalo de 12 horas, era reconduzida à baia do reprodutor, na tentativa de assegurar a prenhez.

O diagnóstico de gestação foi realizado 100 dias após a cobertura, por ultra-sonografia trans-retal. Após a confirmação da prenhez, foram escolhidas, combase na homogeneidade 
do peso vivo, 24 ovelhas para compor as unidades experimentais.

As dietas experimentais, diferenciadas quanto ao nível energético, foram compostas de feno de Tifton 85, farelo de milho, farelo de soja, farelo de algodão, óleo vegetal, palma forrageira, uréia e núcleo mineral ovino, formuladas após análise bromatológica, de forma que a dieta de maior nível possuísse 2,8 Mcal de EM/kg de MS e as demais, 85 e 71\% deste valor, respectivamente (Tabela 1 ).

Após a formação dos grupos, os animais foram alimentados com a respectiva dieta durante todo o terço final da gestação.

Durante a fase experimental, as ovelhas (e suas crias) permaneceram nas baias descritas anteriormente. As dietas foram fornecidas em dois horários (8 e 15 h), com água ad libitum, sendo permitido aos cordeiros acesso irrestrito à teta.

Tabela 1 - Composições percentual e bromatológica das dietas experimentais

Table 1 - Percentage and chemical compositions of the experimental diets

\begin{tabular}{|c|c|}
\hline \multirow[t]{2}{*}{ Item } & $\begin{array}{c}\text { Nível de energia } \\
\text { metabolizável (Mcal/kg MS) } \\
\text { Metabolizable energy level }\end{array}$ \\
\hline & 2,8 \\
\hline
\end{tabular}

Composição percentual (\%MS)

Percentage composition (\%DM)

\begin{tabular}{|c|c|c|c|}
\hline Feno de Tifton-85 (Tifton hay) & 70,00 & 50,00 & 30,00 \\
\hline Palma forrageira (Spineless cactus) & 10,00 & 10,00 & 10,00 \\
\hline Concentrado (Concentrate) & 20,00 & 40,00 & 60,00 \\
\hline Farelo de milho (Corn meal) & 2,00 & 19,00 & 42,70 \\
\hline Farelo de soja (Soybean meal) & 10,50 & 10,00 & 6,00 \\
\hline Farelo de algodão (Cottonseed meal) & 3,50 & 5,00 & 4,00 \\
\hline Uréia (Urea) & 1,00 & 0,50 & 0,50 \\
\hline Óleo de soja (Soybean oil) & 0,00 & 2,50 & 4,80 \\
\hline Calcário calcítico (Limestone) & 1,50 & 1,50 & 1,00 \\
\hline Núcleo mineral (Mineral mixture) ${ }^{1}$ & 1,50 & 1,50 & 1,00 \\
\hline
\end{tabular}

Composição bromatológica (\%MS)

Chemical composition (\%DM)

\begin{tabular}{lrrr}
\hline MS (\%) (DM) & 44,02 & 44,07 & 43,94 \\
MO (\%) (OM) & 87,12 & 88,01 & 90,08 \\
PB (\%) (CP) & 12,50 & 12,50 & 12,50 \\
NDT (\%) (TDN) & 46,00 & 55,00 & 64,00 \\
EM (Mcal/kg MS) (ME) & 2,03 & 2,42 & 2,84 \\
EE (\%) & 1,96 & 4,71 & 7,41 \\
FDN (\%) & 58,92 & 46,78 & 34,21 \\
FDA (\%) (ADF) & 28,80 & 22,71 & 16,20 \\
Ca (g/kg) & 3,67 & 2,97 & 1,94 \\
P (g/kg) & 1,58 & 1,29 & 0,90 \\
Relação Ca:P (Ca:P ratio) & $2,32:$ & $2,31:$ & $2,16:$ \\
& 1,00 & 1,00 & 1,00
\end{tabular}

${ }^{1}$ Núcleo mineral (nutriente/kg de suplemento) (Mineral mix, content per $\mathrm{kg}$ of product): Ca, $190 \mathrm{~g} ; \mathrm{P}, 73 \mathrm{~g} ; \mathrm{Mg}, 44 \mathrm{~g} ; \mathrm{Na}, 62 \mathrm{~g} ; \mathrm{Cl}, 92 \mathrm{~g} ; \mathrm{S}, 30 \mathrm{~g} ; \mathrm{Zn}$, 1.350 mg; Cu, 340 mg; Mn, 940 mg; Fe, 1064 mg; Co, 3 mg; I, 16 mg; Se, $18 \mathrm{mg}$; F máximo (maximum F), $730 \mathrm{mg}$.
A qualidade da relação materno-filial foi avaliada por meio de um estudo comportamental, observando-se as ações durante as duas primeiras horas após o parto, com registros dos eventos mais importantes relativos à aceitação/rejeição em planilha própria. Uma vez detectada uma ovelha com sinais característicos do pré-parto, iniciava-se o acompanhamento, com o registro da data e do número de identificação. A hora exata do parto era estabelecida quando ocorria a expulsão completa do feto e, então, iniciavam-se os registros dos comportamentos da mãe e do neonato.

O método adotado para análise do comportamento materno foi a amostragem focal com registro temporal instantâneo (Dellinger, 2004). O tempo de observação foi subdividido em intervalos de amostragem de cinco minutos cada, totalizando 24 momentos de visualização no decorrer das duas horas; no final de cada intervalo, o animal era observado e a ocorrência ou não do evento pré-estabelecido, registrada.

Os eventos maternos considerados foram a vocalização (OV), o ato de cheirar e lamber a cria (OLCC), a posição da ovelha (em pé - OEP) e a rejeição da cria.

Nas demais variáveis analisadas, que foram o tempo decorrido do nascimento no momento em que o cordeiro ficou de pé pela primeira vez (CPAN), o tempo decorrido do nascimento à primeira mamada (PMAN) e o tempo total de mamadas (TTM), aplicou-se o método de amostragem focal com registro contínuo (Dellinger, 2004).

Os procedimentos de manejo - desinfecção de umbigo, marcação e pesagem - foram efetuados somente após o término dessa avaliação.

Os dados foram analisados empregando-se o teste de contraste de médias por meio do programa computacional SAS (1996).

\section{Resultados e Discussão}

Notou-se efeito da dieta sobre as características comportamentais maternas avaliadas, de modo que, à medida que o aporte energético aumentou, elevou-se o número de ações desenvolvidas durante o período de avaliação (Tabela 2). Estes resultados decorreram, provavelmente, de maior disponibilidade de reservas energéticas das ovelhas que receberam a nutrição mais adequada (2,8 Mcal de EM), ou mesmo por um carreamento de nutrientes obtidos na dieta diretamente para os tecidos fetais e mamário em formação das ovelhas que passaram pela restrição energética. Esta hipótese se fundamenta na variação significativa do escore corporal após o parto das ovelhas que compunham o grupo que receberam dieta com 2,8 Mcal de EM/kg MS em relação aos demais tratamentos. Consideran- 
Tabela 2 - Características comportamentais maternas, em função do nível de EM na dieta

Table 2 - $\quad$ Characteristics of maternal behavior according to the dietary ME level

\begin{tabular}{lccrr}
\hline Item & \multicolumn{3}{c}{$\begin{array}{c}\text { Nível de EM na } \\
\text { dieta (Mcal/kg MS) }\end{array}$} & CV (\%) \\
& \multicolumn{2}{c}{\begin{tabular}{c} 
ME level in the diet (Mcal/kg DM) \\
\cline { 2 - 4 }
\end{tabular}} & & \\
\cline { 2 - 4 } & 2,0 & 2,4 & 2,8 & \\
\hline OEP (n) (SSU, $n)$ & $23,10 \mathrm{~b}$ & $23,94 \mathrm{a}$ & $23,64 \mathrm{a}$ & 2,55 \\
OLCC (n) (SLSL, $n)$ & $13,40 \mathrm{c}$ & $19,94 \mathrm{~b}$ & $21,09 \mathrm{a}$ & 28,37 \\
OV (n) $(S V, n)$ & $15,30 \mathrm{c}$ & $19,10 \mathrm{~b}$ & $21,91 \mathrm{a}$ & 25,68 \\
\hline
\end{tabular}

OEP - ovelha em pé; OLCC - ovelha lambendo e cheirando o cordeiro; OV - ovelha vocalizando.

Médias seguidas de letras diferentes na mesma linha diferem $(P<0,05)$ pelo teste de contraste de médias.

SSU - sheep stand up; SLSL - sheep licking and smelling the lamb; SV - sheep vocalizing. Means followed by different letters within a row are different $(P<0.05)$ by test of contrast of means.

$\mathrm{n}=$ número de vezes ( $n=$ number of times).

do a escala de 1 a 5 para escore corporal, Torreão (2007) identificou nos mesmos animais desta pesquisa valores de 2,8; 2,5 e 2,25 para as ovelhas alimentadas com as dietas de níveis máximo, médio e mínimo de EM, respectivamente.

Ullrey et al. (1970) concluíram que o não atendimento das exigências da ovelha gestante forçaria o carreamento dos depósitos de gordura para prover o déficit de energia, mas em uma dieta completamente inadequada poderia sacrificar, inclusive, os ossos e tecidos do corpo para nutrir o feto. Na variável OEP, entretanto, a diferença não se mostrou significativa entre os níveis médio e máximo de EM das dietas deste experimento.

Os efeitos da nutrição durante o período gestacional sobre o comportamento materno da ovelha foram demonstrados por Thomson \& Thomson (1949), trabalhando com ovelhas da raça Merina, e Putu et al. (1988), com ovelhas da raça Cerf de Viginie. Em ambos os trabalhos, utilizaram-se dois grupos de animais, um recebendo alimentação ad libitum e outro com restrição energética, resultando em ausência de comportamento materno nas fêmeas mal-alimentadas, com conseqüente aumento na taxa de mortalidade. Esses autores atribuíram este comportamento à ação da alimentação. As fêmeas que foram mal nutridas durante a gestação não dispunham de reservas energéticas satisfatórias e, por isso, ficaram mais debilitadas, em decorrência dos esforços realizados durante a parição para a expulsão do feto, comprometendo sua habilidade materna. Langeneau \& Lerg (1976) confirmaram esse fato ao demonstrarem os efeitos negativos da pastagem de baixa qualidade sobre o comportamento materno e o posterior desenvolvimento das crias ovinas.

Embora tenham apresentado menores médias que as ovelhas do tratamento com melhor aporte energético no experimento, os animais que compuseram as parcelas dos
Tabela 3 - Características comportamentais filiais, em função do nível de EM na dieta

Table 3 - Characteristics of filial behavior according to the dietary ME level

\begin{tabular}{lcccc}
\hline Item & \multicolumn{3}{c}{$\begin{array}{c}\text { Nível de EM na } \\
\text { dieta (Mcal/kg MS) }\end{array}$} & CV (\%) \\
& \multicolumn{2}{c}{ ME level in the diet (Mcal/kg DM) } & \\
\cline { 2 - 3 } & 2,0 & 2,4 & 2,8 & \\
\hline CEP(n) & 13,00 & 14,20 & 16,20 & 40,51 \\
$\begin{array}{l}\text { LSU } \\
\text { CPAN (min) }\end{array}$ & 9,90 & 19,68 & 9,90 & 143,03 \\
$\begin{array}{l}\text { LSUAB (min) } \\
\text { PMAN (min) }\end{array}$ & 39,70 & 55,80 & 44,09 & 60,83 \\
$\begin{array}{l}\text { FSAB (min) } \\
\text { TTM (min) } \\
\text { TTS (min) }\end{array}$ & 2,28 & 3,08 & 2,84 & 86,45 \\
\hline
\end{tabular}

CEP - cordeiro em pé; CPAN - cordeiro de pé após o nascimento, PMAN - primeira mamada após o nascimento; TTM - tempo total de mamadas. LSU - lamb stand up, LSUAB - lamb stand up after birth, FSAB - first suckling after birth; TTS - total time suckling.

* Não-significativo $(P>0,05)$ (Not significant - $P>0.05)$.

$\mathrm{n}=$ número de vezes ( $n=$ number of times).

$\min =$ minutos $(\min =$ minutes $)$

níveis mínimo e médio não deixaram de expressar seus cuidados, nem tampouco chegaram a rejeitar suas crias. Este resultado pode ser explicado pela pressão de seleção natural imposta à raça, que permitiu evidenciar ótima habilidade materna, mesmo em condições de restrição energética.

No estudo dos parâmetros comportamentais dos cordeiros, não se notou diferença entre os tratamentos, fato provavelmente atribuído à capacidade de carreamento nutricional das ovelhas para os fetos, que gerou pesos ao nascer de 2,38; 2,25 e 2,53 kg para os níveis de 2,0, 2,4 e 2,8 Mcal de EM/kg MS respectivamente, sem apresentar diferença significativa $(\mathrm{P}>0,05)$ (Tabela 3$)$. Essa faixa se mostra intermediária à observada na literatura para essa raça: Oliveira (1992) e Lôbo et al. (1992) obtiveram, respectivamente, média de 2,79 e 1,82 kg. Portanto, todos os animais, independentemente do tratamento a que foram submetidos, estavam em condições corporais pós-parto semelhantes e satisfatórias e, por esse motivo, tiveram a possibilidade de apresentar o mesmo padrão de comportamento, uma vez que suas mães, embora tenham sofrido efeitos dos tratamentos, não demonstraram comportamento de rejeição e desempenharam um cuidado maternal que estabeleceu o vínculo concreto com suas crias e as estimulou a se comportarem satisfatoriamente.

\section{Conclusões}

Mesmo sob interferência das ações de relacionamento com as crias, as ovelhas da raça Morada Nova conseguiram manter os neonatos em semelhantes condições, quando submetidas a dietas de menores níveis de energia. 


\section{Agradecimento}

Ao FUNDECI/ETENE/BNB, pelo financiamento da pesquisa, e a todos que contribuíram para o desenvolvimento deste trabalho.

\section{Literatura Citada}

ALEXANDER, G.; SIGNORET, J.P.; HAFEZ, E.S.E. Sexual and maternal behavior. In: HAFEZ, E.S.E. (Ed.), Reproduction in farm animals. 3.ed. Philadelphia: Lea \& Febiger, 1974. p.222-254.

BROWN, R.E. Hormônios e comportamento parental. In: COSTA, M.J.R.P.; CROMBERG, V.U. (Eds.). Comportamento materno em mamíferos (bases teóricas a aplicações aos ruminantes domésticos). São Paulo: Sociedade Brasileira de Etologia, 1998. p.53-99.

COSTA, M.J.R.P.; CROMBERG, V.U. Comportamento materno em mamíferos, bases teóricas e aplicações aos ruminantes domésticos. São Paulo: Sociedade Brasileira de Etologia, 1998. 272p.

CUnha, E.A.; SANTOS, L.E.; Bueno, M.S. et al. Produção intensiva de ovinos. Nova Odessa: Instituto de Zootecnia, 1999. 49p.

Dellinger, F.T.U. Programa, conteúdos e métodos de ensino teórico e prático da disciplina de Etologia. Lisboa: Universidade da Madeira, 2004. 118p.

FERREL, C.L. Nutrient requirements, other factors affect fetal growth. Feedstuffs, v.17, p.18-41, 1992.

GERASEEV, L.C.; PEREZ, J.R.O.; CARVALHO, P.A. et al. Efeitos das restrições pré e pós-natal sobre o crescimento e o desempenho de cordeiros Santa Inês do nascimento ao desmame. Revista Brasileira de Zootecnia, v.35, n.1, p.245-251, 2006.

JOCHIMS, K.; KAUP, F.J.; DROMMER, W. et al. An immunoelectron microscopic investigation of colostral IgG absorption across the intestine of newborn calves. Research Veterinary Science, v.57, p.75-80, 1994.

KELLER, M.; MEURISSE, M.; POINDRON, P. et al. Maternal experience influences the establishment of visual/auditory, but not olfactory recognition of the newborn lamb by ewes at parturition. Developmental Psychobiology, v.43, n.3, p.167176, 2003.

LANGENAU, E.E.; LERG, J.M. The effects of winter nutritional stress on maternal and neonatal behavior of penned whitetailed deer. Applied Animal Ethology, v.2, p.207-223, 1976.

LEVY, F.; KELLER, M.; POINDRON, P. Olfactory regulation of maternal behavior in mammals. Hormones and Behavior, v.46, n.3, p.284-302, 2004.

LÔBO, R.N.B.; MARTINS FILHO, R.; FERNANDES, A.A.O. Efeitos de fatores genéticos e de ambiente sobre o peso ao nascimento de ovinos da raça Morada Nova no sertão do Ceará. Ciência Animal, v.2, n.1, p.95-104, 1992.
MELLOR, D.J. Nutritional effects on the fetus and mamary gland during pregnancy. Proceedings of Nutrition Society, v.46, p.249-257, 1987.

OLIVEIRA, S.M.P.; MIRANDA, J.J.F.; PEREIRA, C.S. et al. Parâmetros genéticos e efeitos não genéticos dos pesos ao nascer, aos 112, aos 210 e aos 365 dias de idade de ovinos da raça Morada Nova variedade branca. In: REUNIÃO ANUAL DA SOCIEDADE BRASILEIRA DE ZOOTECNIA, 29., 1992, Lavras. Anais... Lavras: Sociedade Brasileira de Zootecnia, 1992. p.95.

PINHEIRO, L.C.; MACHADO, F.; HURNIK, J.F. et al. Timing of the attraction towards the placenta and amniotic fluid by the parturient cow. Applied Animal Behavior Science, v.53, p.185-194, 1997.

POINDRON, P.; LEVY, F.; KREHBIEL, D. Genital, olfactory, and endocrine interactions in the development of maternal behaviour in the parturient ewe. Psychoneuroendocrinology, v.13, p.99-125, 1988.

POINDRON, P.; NOWAK, R.; LEVY, F. et al. Development of exclusive bonding in sheep and goats. Oxford Reviews of Reproductive Biology, v.15, p.311-364, 1993.

PUTU, I.G.; POINDRON, P.; LINDSAY, D.R. A high level of nutrition during late pregnancy improves subsequent maternal behaviour of merino ewes. Proceedings Australian Society Animal Production, v.17, p.294-297, 1988.

RAMIREZ, A.; QUILES, A.; HEVIA, M.L. et al. Influence of forced on the maternal-filial bond in the domestic goat after different periods of post-partum separation. Small Ruminant Research, v.23, p.75-81, 1996.

SILVA SOBRINHO, A.G.; BATISTA, A.M.V.; SIQUEIRA, E.R. et al. Nutrição de ovinos. Jaboticabal: FUNEP, 1996. p.258.

STATISTICAL ANALYSIS SYSTEM - SAS. User's guide. Cary: 1996. 842p.

SCHAAL, B.; ORGEUR, P.; ARNOULD, C. Olfactory preferences in newborn lambs: possible influence of prenatal experience. Behaviour, v.132, p.251-263, 1995.

THOMSON, A.M.; THOMSON, W. Lambing in relation to the diet of the pregnant ewe. British Journal Nutrition, v.2, p.290305, 1949.

TORREÃO, J.N.C. Ingestão de energia em ovelhas Morada Nova no terço final da gestação e no pós-parto. Areia: Universidade Federal da Paraíba, 2007. 213p. Tese (Doutorado em Zootecnia) - Universidade Federal da Paraíba, 2007.

VINCE, M.A. Newborn lambs and their dams: the interactions that lea to sucking. Advances in the Study of Behavior, v.22, p.239-268, 1993.

ULLREY, D.E.; YOUATT, W.G.; JOHNSON, H.E. et al. Digestible and metabolizable energy requirements for winter maintenance of Michigan white-tailed does. Journal of Wildlife Management, v.34, n.4, p.863-869, 1970. 